\title{
EIN(FACH)? Komplexität, Wissen, Fortschritt und die Grenzen der Germanistik
}

\author{
Klaus-Michael Bogdal (Bielefeld)
}

»Man ist nicht umsonst Philologe gewesen «.

Friedrich Nietzsche: Morgenröthe

»5 910,29 EURO «

Grundgehalt C 4, Stufe 15

\section{Einleitung: Komplexität und Fortschritt}

Spätestens seit den gesellschaftlichen Modernisierungsschüben ${ }^{1}$ in den sechziger Jahren identifiziert auch die Germanistik Erkenntnis- und Wissenszuwachs, ja allgemeiner den >Fortschritt< ihres Fachs, mit Komplexitätserhöhung. Vor diesem Hintergrund erscheint es mir wenig plausibel, die seitdem erfolgten inneren Ausdifferenzierungen und interdisziplinären Grenzüberschreitungen als durch Identitätsverlust, Zerstreuung 2 und Desintegration gekennzeichnete Niedergangsszenarien zu beschreiben. ${ }^{3}$ Die Veränderungen gehorchen der immanenten Logik germanistischer Forschung, einer >disziplinierten<, auf Leistung ausgerichteten, an kooperativen Großforschungsvorhaben partizipierenden Wissensproduktion. ${ }^{4}$

Wenn seitdem immer wieder gefragt wird, »ob eine >Einheit< des Faches überhaupt realistisch oder auch nur wünschenswert ist « ${ }^{5}$, so muss zunächst zurückgefragt werden, welcher Typus von Einheit überhaupt gemeint ist. Das Schlagwort der Re-Philologisierung weist recht ungenau auf die Anfänge des Fachs vor dem ersten gravierenden Wandel wissenschaftlicher Organisation

1 Vgl. Sill, Oliver: Kein Ende und ein Anfang: germanistische Literaturwissenschaft der sechziger und siebziger Jahre. Bielefeld 2003 und Herbert, Ulrich (Hg.): Wandlungsprozesse in Westdeutschland. Belastung, Integration, Liberalisierung 19451980. Göttingen 2002.

2 Vgl. Osterkamp, Ernst: »Medien der Germanistik: Anspruch und Praxis literaturwissenschaftlichen Publizierens. Vorbemerkung zu einer Diskussion«. In. Jahrbuch der deutschen Schillergesellschaft 45 (2001), S. 1-7, der die gegenwärtige Fachkommunikation als desintegrierend beschreibt. Vgl. dagegen Röcke, Werner: »Interdisziplinarität und >Einheit< des Fachs. Ein Plädoyer für die >Zerstreung< in der Altgermanistik.« In: Jahrbuch der deutschen Schillergesellschaft 46 (2002), S. 342348.

3 Hier Vietta, Silvio/Kemper, Dirk (Hg.): Germanistik der 70er Jahre. Zwischen Innovation und Ideologie. München 2000.

4 Vgl. Fohrmann, Jürgen/Voßkamp, Wilhelm (Hg.): Wissenschaft und Nation. Zur Entstehungsgeschichte der deutschen Literaturwissenschaft. München 1991.

5 Osterkamp: Medien der Germanistik (s. Anm. 2), S. 5. 
und geisteswissenschaftlicher Methoden um 1880 zurück. Um die Frage nach den Grenzen der Germanistik ernsthaft erörtern zu können, genügt es keinesfalls, sich vornehmlich auf die große Identitätskrise der 1960er und 70er zu beziehen, in der eine sich explosionsartig ausbreitende Massenuniversität die nicht zu bestreitende Diversifizierung des Fachs begünstigte. Die neuere Fachgeschichtsschreibung hat zeigen können, dass schon in der Zeit um 1880 ein enger Zusammenhang zwischen der Veränderung »in Struktur und Organisation wissenschaftlichen Wissens $\ll^{6}$, hier genauer der Gründung zahlreicher Germanistischer Seminare und damit der Etablierung einer Universitätsdisziplin mit einer gewissen Bandbreite, ${ }^{7}$ und dem Aufkommen konkurrierender Forschungsprogramme zu beobachten ist. Diese veränderte Situation löst »eine bis dahin nicht gekannte Instabilität des disziplinären Selbstverständnisses $\ll^{8}$ aus. Es sind vor allem interne philologische Probleme »einer selbst produzierten unbestimmten Komplexität « ${ }^{9}$ der Scherer-Schule, die zur Kritik der noch philologisch sozialisierten ersten Generation geisteswissenschaftlicher Germanisten an die Grundlagen ihres Fachs führen, denn deren Arbeiten genügen dem Anspruch, an der Schaffung eines nationalen Mythos mitzuwirken, nicht. ${ }^{10} »$ Textkritik, Edition, Quellen- und Einflussforschung, Stoffgeschichte, Entstehungsgeschichte, im einzelnen noch Metrik und Poetik «11, gelten unter den neuen Bedingungen und Möglichkeiten als Ausdruck eines unterkomplexen Fachverständnisses, das einer entschiedenen, aus damaliger Perspektive geistesgeschichtlichen, Erweiterung bedurfte. ${ }^{12}$

Mit den gegenwärtigen Rufen nach Re-Philologisierung wird, wenn nicht ein Versprechen, so doch eine Hoffnung artikuliert: die Wiedergewinnung germanistischer Identität und disziplinärer Einheit. Bevor diese Hoffnung wieder enttäuscht wird, sollte in guter philologischer Tradition danach gefragt werden, ob eine solche Philologie in der Germanistik jemals konkrete Gestalt angenommen hat. Ich habe da erhebliche Zweifel. Trägt nicht im

6 Wegmann, Nikolaus: »Philologische Selbstreflextion. Die Frage nach der disziplinären Einheit.«In: Fohrmann/Voßkamp: Wissenschaft und Nation (s. Anm. 4), S. 118.

7 Kolk, Rainer: »Reflektionsformel und Ethikangebot.«In: König, Christoph/Lämmert, Eberhard (Hg.): Literaturgeschichte und Geistesgeschichte 1910-1925. Frankfurt a. M. 1993, S. 38-45, hier S. 39.

8 Kolk: Reflektionsformel und Ethikangebot (s. Anm. 7), S. 38.

9 Dainat, Holger: »Überbietung der Philologie«. In: König/Lämmert: Literaturgeschichte und Geistesgeschichte 1910-1925 (s. Anm. 7), S. 232-239, hier S. 233.

10 Vgl. Weimar, Klaus: »Das Muster geistesgeschichtlicher Darstellung. Rudolf Ungers Einleitung zu >Hamann und die Aufklärung««. In: König/Lämmert: Literaturgeschichte und Geistesgeschichte 1910-1925 (s. Anm. 7), S. 92-105.

11 Barner, Wilfried: »Zwischen Gravitation und Opposition. Philologie in der Epoche der Geistesgeschichte.«In: König/Lämmert: Literaturgeschichte und Geistesgeschichte 1910-1925 (s. Anm. 7), S. 201-231, hier S. 204.

12 Kolk: Reflektionsformel und Ethikangebot (s. Anm. 7), S. 40: »Die Relativierung der Philologie soll deren reduziertes Wissenschaftsverständnis ersetzen durch ein komplexeres, das jener Praxis ihren Ort zuweist, ihre Legitimität begründet, aber auch begrenzt.« 
Gegenteil besonders die deutsche Philologie, die um 1800 als eine der ersten modernen Humanwissenschaften entsteht, die Notwendigkeit ständiger Komplexitätserhöhung in sich, weil auch in ihr das Subjekt selbst zum Objekt der Erkenntnis gemacht wird? ${ }^{13}$ Und ist nicht durch die historisch-anthropologischen Implikationen die Dynamik der >Grenzüberschreitungen< bis hin zur Selbstaufhebung >im Leben< (der Nation) von Anfang an wirksam? Nach Foucaults Beobachtung ist der »Gegenstand der Humanwissenschaften nicht die [...] Sprache, es ist jenes Wesen, das vom Innern der Sprache, durch die es umgeben ist, sich beim Sprechen den Sinn der Wörter oder der von ihm ausgesprochenen Sätze repräsentiert und schließlich die Repräsentation der Sprache selbst gibt. «14 Diese >Dopplung<, die dazu zwingt, sowohl den Gegenstand mit jedem Wissensfortschritt zu erweitern und neu zu definieren, als auch die Subjektposition jeweils neu zu bestimmen, führt zu einer nicht reversiblen Entwicklung in der deutschen Germanistik vom Gelehrtentum zur » disziplinäre(n)< Gemeinschaft «15.

Die zahlreichen >Überbietungen< der Philologie, die seit ungefähr 1880 die Germanistik ständig verändert haben, resultieren immer noch aus den humanwissenschaftlichen Anfängen. Einzig die Entstehung der modernen Linguistik in den 1960ern könnte mit guten Argumenten als radikaler, wissenschaftstheoretisch begründeter Bruch mit der philologischen Tradition gedeutet werden. Eine solche Deutung hätte allerdings zur Folge, dass die Linguistik im Blick auf die disziplinäre Einheit als nicht re-integrierbar betrachtet werden müsste. Was die anderen Transformationen betrifft, die sich im Laufe der langen Geschichte des Fachs ereignet haben, so hat Wilfried Barner exemplarisch am Verhältnis der geisteswissenschaftlichen Germanistik zur philologischen Tradition dargelegt, dass diese die philologischen Grundbestände niemals veräußert hat. ${ }^{16}$

Mit dem Hinweis auf die humanwissenschaftlichen Anfänge der Germanistik im Wissensdispositiv um 1800 möchte ich in Erinnerung bringen, dass die Frage nach der Einheit des Fachs und nach den Grenzen der Disziplin sich nicht von den jeweiligen Komplexitätszuschreibungen trennen lässt. Wir blicken auf eine nun gut zweihundertjährige Geschichte variabler, verhandelbarer und umstrittener wissenschaftlicher Konstruktionen von Literatur und Sprache zurück, die zudem dem Wandel des allgemeinen Wissenschaftsverständnisses ausgesetzt waren. Folgt man Luhmanns Theorie der »Kunst der Gesellschaft «17, die behauptet, dass Kunstwerke wegen der spezifischen, >paradoxen< Komplexität ihrer Entstehung »als Einheit nicht beobachtet« und

13 »Wo sind Sie, Professor Foucault? (Gespräch mit P. Caruso). In: Foucault, Michel: Schriften in vier Bänden. Dits et Ecrits. Bd. I. 1954-1969. Frankfurt a. M. 2001, S. 770-793, hier S. 778: »als Subjekt jeglichen Wissens und als Objekt eines möglichen Wissens «.

14 Foucault, Michel: Die Ordnung der Dinge. Eine Archäologie der Humanwissenschaften. Frankfurt a. M. 1974, S. 423.

15 Fohrmann/Voßkamp: Wissenschaft und Nation (s. Anm. 4), S. 11.

16 Vgl. Barner: Zwischen Gravitation und Opposition (s. Anm. 11).

17 Luhmann, Niklas: Die Kunst der Gesellschaft. Frankfurt a. M. 1995. 
damit auch »nicht beschrieben werden « können, ${ }^{18}$ so müsste die philologische Wissenschaft, der es programmatisch um die Totalität der Hervorbringungen und Überlieferungen von Literatur geht, an der Besonderheit ihres Gegenstandes scheitern. Man muss Luhmanns Annahmen zur Kunst nicht teilen - und ich teile sie nicht -, die modernisierungstheoretischen Überlegungen, die ihnen zu Grunde liegen, lassen sich kaum von der Hand weisen.

Was bedeutet unter der modernisierungstheoretischen Prämisse, dass jede Theorie, die Anspruch auf allgemeine Anerkennung erhebt, dazu in der Lage sein muss, die Komplexität ihres Gegenstands zu erfassen, ${ }^{19}$ für die Idee einer Re-Philologisierung der Germanistik?

Man kann an Hans Ulrich Gumbrechts Programmschrift Die Macht der Philologie beobachten, wie dieser entscheidende Punkt mit »special effects $« 20$ (um einen der präzisen Fachtermini des Autors aufzugreifen) abgedunkelt wird. Wohin genau soll das Präfix zurückführen? $\mathrm{Zu}$ den Wurzeln der Deutschen Philologie im frühen neunzehnten Jahrhundert, wie bei Gumbrecht zunächst angedeutet und dann wieder durchgestrichen; zur auratischen geistesgeschichtlichen Germanistik Gundolfs, von der das gehobene deutsche Feuilleton träumt; zu einer um die avantgardistische Moderne und die Kritische Theorie angereicherten Nachkriegsgermanistik wie bei Bohrer; zur New Philology; oder, so Gumbrechts Wunschvorstellung, zu einem »neuen intellektuellen Stil « ${ }^{21}$ ohne Forschungsprogramm, aber mit einer Lizenz zum Mitreden in der Welt?

Die Re-Philologisierung verspricht, wenn man die bisher noch vagen und in unterschiedliche Richtungen weisenden Ansätze zusammennimmt, drei Veränderungen:

- die Wiederherstellung einer Kommunikationsgemeinschaft bzw. eines Bildungsmilieus der Wissenden,

- die Bestimmung und Kanonisierung wesentlicher, >eminenter< Gegenstände 22 ,

- die Verlangsamung der Fachdynamik insbesondere im Blick auf Methoden und auf transdisziplinäre Vernetzung.

Die Versprechungen richten sich an die beteiligten Subjekte und rufen deren Unbehagen an der universitären Praxis in der Gegenwart auf. Sie führen nicht zur Philologie, sondern zum faszinierenden Bild des integren Kunstkenners zurück, dass der Philologenverächter Nietzsche in einem seiner berühmtesten Morgenröthe-Fragmente entworfen hat:

18 Luhmann: Die Kunst der Gesellschaft (s. Anm. 17), S. 74 f.

19 Vgl Binczek, Natalie: »Zur Funktion des Ornaments in Luhmanns Kunst-Buch«. In: Schwering, Gregor/Zelle, Carsten (Hg.): Ästhetische Positionen nach Ardorno. München 2002, S. 1103-1122, hier S. 1112.

20 Gumbrecht, Hans Ulrich: Die Macht der Philologie. Frankfurt a. M. 2003, S. 20.

21 Gumbrecht: Die Macht der Philologie (s. Anm. 20), S. 20.

22 Vgl. Schlaffer, Heinz: Die kurze Geschichte der deutschen Literatur. München 2002 . 
»Philologie nämlich ist jene ehrwürdige Kunst, welche von ihrem Verehrer vor Allem Eins heischt, bei Seite gehn, sich Zeit lassen, still werden, langsam werden-, als eine Goldschmiedekunst und -kennerschaft des Worte s, die lauter feine, vorsichtige Arbeit abzuthun hat und Nichts erreicht, wenn sie es nicht lento erreicht. Gerade damit ist sie aber heute nöthiger als je, gerade dadurch zieht sie und bezaubert sie uns am stärksten, mitten in einem Zeitalter der >Arbeit<, will sagen: der Hast, der unanständigen und schwitzenden Eilfertigkeit, das mit Allem gleich >fertig werden will, auch mit jedem alten und neuen Buche: - sie selbst wird nicht so leicht irgend womit fertig, sie lehrt gut lesen, das heisst langsam, tief, rück- und vorsichtig, mit Hintergedanken, mit offen gelassenen Thüren, mit zarten Fingern und Augen lesen ...«23

Die Idee einer Rückkehr zur Philologie schließt - angesichts der als Entgrenzung wahrgenommenen kulturwissenschaftlichen Wende in sämtlichen Teilfächern der Germanistik, d.h. ebenfalls in der Mediävistik/Altgermanistik und in der Angewandten Linguistik und Sprachgeschichte - die Annahme ein, dass es vor der sogenannten Reformperiode der 1960er und 1970er, die durch beschleunigte institutionelle Modernisierungen, fachliche Innovationen und interdisziplinäre Grenzüberschreitungen gekennzeichnet war, ${ }^{24}$ Entwicklungsphasen gegeben habe, in denen die drei oben genannten Bedingungen erfüllt waren und eine für die Beteiligten erfahrbare Einheit existierte, die wiederum $>$ Identität< erzeugte.

Der Idee der Re-Philologisierung möchte ich einige, in eine andere Richtung weisende Überlegungen entgegenstellen, die sich in erster Linie auf wissenschaftsgeschichtliche Beobachtungen stützen. Wie schon angedeutet, wird die Deutsche Philologie als moderne Humanwissenschaft geboren. Damit gehört sie zu einem Wissenschaftstyp, der um den Preis des Verschwindens durch jede zu einem bestimmten Zeitpunkt als > wahr $<$ akzeptierte Entdeckung über das Wesen des Menschen (von der Evolutionstheorie über das Unbewusste bis zur Kognition) zu einer Evaluierung der eigenen Wissensbestände gezwungen wird. Als universitäres Fach, das an seiner >Leistung < gemessen wird, folgt sie zudem der Dynamik institutioneller Wissenschaft - bis hin zu rassistischen Exzessen, die nicht als Konsequenz externen Drucks, sondern interner Logiken entstanden sind. Ein >Zurück < selbst zu den Wurzeln befreit deshalb nicht vom Zwang zum wissenschaftlichen Fortschritt.

Der Germanistik ist es im Verlauf ihrer Entwicklung - trotz avancierter Vorstöße um 1880, in den zwanziger und sechziger Jahren - bisher nicht gelungen, einen Typus von Einheit zu finden, der nicht wieder zyklisch durch die interne Dynamik aufgesprengt würde. Das spricht nicht unbedingt gegen sie, denn nicht jede Innovation bedeutet Niedergang. Gemeint ist ein Typus, der

23 Nietzsche, Friedrich: »Morgenröthe. Nachgelassene Fragmente. Anfang 1880 bis Frühjahr 1881.«In: Nietzsches Werke. Bd. V.1. Hrsg. von Giorgio Colli und Mazzino Montinari. Berlin 1971, S. 9.

24 Vgl. Bogdal, Klaus-Michael/Müller, Oliver (Hg.): Innovation und Modernisierung. Germanistik von 1965 bis 1980 (in Vorb.). 
Kohärenz stiftet und Kommunikation garantiert, der Neuentwicklungen nicht behindert und den Subjekten Identifikationen erlaubt und der vielleicht auch noch für Nietzsches Erotik des Lesens mit >zarten Fingern< Raum lässt.

Nach meiner Überzeugung besitzen wir inzwischen genug Erfahrung und Reflexionswissen, um einen solchen Typus - auch im Blick auf die Organisation des Wissens und die institutionellen Bedingungen - zu denken: ein Gegenmodell zur Entropie einer monoparadigmatischen Germanistik gleich welchen theoretischen und methodischen Zuschnitts.

\section{Wissen und Rückschritt}

Die Germanistik durchlief zwischen 1910 und 1930 einen deutlichen Aufschwung und eine erhebliche Ausdifferenzierung zu einer Disziplin zur Erforschung des deutschen Geistes. ${ }^{25} \mathrm{Ihr}$ widersprüchliches Verhältnis zur philologischen Tradition hat Wilfried Barner ausführlich dargestellt. ${ }^{26}$ Nach dieser Phase und unter den besonderen Bedingungen der Verdrängung der eigenen nationalsozialistischen Vergangenheit ${ }^{27}$ fand in der Nachkriegszeit zwischen ca. 1947 und 1960 eine Bewegung statt, die man, trotz des bisweilen existentialontologischen Überbaus, wegen ihres Zugs »ins Puristische « 28 oder ihrer Tendenz zum »Minimalismus $« 29$ als Re-Philologisierung bezeichnen könnte. Unter dem (späteren) Leitbegriff der Werkimmanenz wurde mit Blick auf die vorangegangenen geistes- und stammesgeschichtlichen Grenzüberschreitungen die Beschränkung auf das Wesentliche/Eigentliche, auf das Kunstwerk, gefordert und praktiziert. Die Nachkriegsgermanistik entwickelte, nicht nur um die Spuren der nationalsozialistischen Vergangenheit zu tilgen, erfolgreiche Strategien der Diskursverknappung und Komplexitätsreduktion. Doch die Formel, dass die Einfachheit die Facheinheit garantiert, funktionierte trotz der günstigen institutionellen Bedingungen, unter denen das überschaubare, hierarchisch strukturierte Fach arbeitete, nicht.

Die germanistische Literaturwissenschaft hielt bis zur werkimmanenten Interpretation die Komplexität der Dichtung und die geistige Schöpferkraft der Dichter für unüberbietbar ${ }^{30}$ und bemühte sich um einen Wissenschaftsstil

25 Vgl. König/Lämmert: Literaturgeschichte und Geistesgeschichte 1910-1925 (s. Anm. 7).

26 Barner: Zwischen Gravitation und Opposition (s. Anm. 11).

27 Vgl. Barner, Wilfried/König, Christoph (Hg.): Zeitenwechsel. Germanistische Literaturwissenschaft vor und nach 1945. Frankfurt a. M. 1996.

28 Hermand, Jost: Synthetisches Interpretieren. Zur Methodik der Literaturwissenschaft. München 1968, S. 8.

29 Bogdal, Klaus-Michael: »Wissenskanon und Kanonwissen. Literaturwissenschaftliche Standardwerke in Zeiten disziplinären Umbruchs. «In: Literarische Kanonbildung. Text und Kritk. Sonderband. München 2002, S. 55-89, hier S. 62.

30 Gadamer spricht in »Wahrheit und Methode« von »Eminenz«; vgl. Gadamer, Hans-Georg: Wahrheit und Methode. Grundzüge einer philosophischen Hermeneutik. Tübingen 1975. 
der gebildeten - nicht der gelehrten - Unterbietung. Aus dieser Haltung heraus, die man als geisteswissenschaftliches Ethos betrachten kann, war es unproblematisch, sich mit einfachen Beschreibungsmodellen ${ }^{31} \mathrm{zu}$ begnügen und auf Bildung und Belesenheit, d.h. auf ein gutes Gedächtnis und die Forscherpersönlichkeit, zu setzen. Die wissenschaftliche Praxis konnte auf wenige übersichtliche >Einheiten< reduziert werden: auf das Werk als singuläre Erscheinung oder als Teil des Gesamtschaffens eines Dichters, auf die AutorWerk-Relation und schließlich - in den Fünfzigern nicht unumstritten - auf die Erzählung einer nationalen Geschichte der Literatur und ihrer geistesgeschichtlichen Dimensionen. Das Reallexikon der deutschen Literaturgeschichte von 1958 definiert »das dichterische Werk als Ausdruck eines bedeutungsmäßig geformten Erlebens von historischer Bedingtheit « und benennt »Autor, Werk und allgemeine Prinzipien (Stil, Gattung, Form, Geist, Problem, Idee) [...] als zentrale Interpretationsziele «.32 In einem für die Zeitschrift Studium Generale von Erich Trunz verfassten Aufsatz, der $1952^{33}$ einem breiten akademischen Publikum eine besondere Merkwürdigkeit der Germanistik, das Interpretieren, als wissenschaftliche Tätigkeit nahe bringen soll, geht es in mehreren argumentativen Anläufen um den Nachweis, dass diese das literarische Werk nicht an Einsichten und Erkenntnissen überbiete. Die erlaubte Nähe zur Dichtung wird als Gefahr und die Interpretation als Risiko erlebt, die durch das >Werk < gesetzte Grenze doch noch zu überschreiten. Die Interpretation gilt Trunz daher als »das Verantwortungsvollste und wohl auch Schwerste in der Philologie. «34 Das Werk ist »ein feststehender Text«, »Dichtung [...] bringt die Zeit zum Stehen «35. Die Interpretation fällt dem Philologen erst durch die Entwicklung der Gesellschaft zur Moderne als Aufgabe $\mathrm{zu}, 36$ in der >das eigene Volk< den unmittelbaren Bezug zu seinem geistigen Erbe zu verlieren droht. »Interpretation ist unnötig für den, welchem eine Dichtung zum Besitz geworden ist. «37 Insofern ist sie für Trunz »immer etwas

31 Diese Beschreibung impliziert keine Wertung der einzelnen Forschungsleistungen.

32 Rappl, Hans Georg: »Interpretation«. In: Kohlschmidt, Werner/Mohr, Wolfgang (Hg.): Reallexikon der deutschen Literaturgeschichte. Erster Band. 2. Aufl. Berlin 1958, S. 752-756, hier S. 755.

33 Holger Dainat hat mich darauf aufmerksam gemacht, dass der Vortrag über Interpretation für den Würzburger Germanistentag 1942 geplant war, der dann kriegsbedingt abgesagt wurde; vgl. Dainat, Holger: »>wir müssen ja trotzdem weiter arbeiten.< Die Deutsche Vierteljahrsschrift für Literaturwissenschaft und Geistesgeschiche vor und nach 1945.« In: Barner/König: Zeitenwechsel (s. Anm. 27), S. 76-100.

34 Trunz, Erich: »Über das Interpretieren deutscher Dichtung «. In: Studium Generale 2 (1952), S. 65-68, hier S. 68.

35 Trunz: Über das Interpretieren deutscher Dichtung (s. Anm. 34), S. 65.

36 Trunz gibt einen wichtigen Hinweis auf den organisatorischen Aspekt, dem ich gern an anderer Stelle nachgehen möchte: »Die moderne Interpretation hat sich entwickelt im akademischen Unterricht, in den literaturwissenschaftlichen Seminaren.«Siehe Trunz: Über das Interpretieren deutscher Dichtung (s. Anm. 34), S. 68.

37 Trunz: Über das Interpretieren deutscher Dichtung (s. Anm. 34), S. 65. 
Sekundäres, Mittelbares, nur ein Hilfsmittel«38. Die »Wissenschaftlichkeit besteht darin, daß sie die menschliche Einseitigkeit durch systematische Umsicht möglichst weitgehend zu überwinden versucht. «39 Der Mikroanalyse wird der Begriff der Werkstruktur zu Grunde gelegt und mit ihm zwei einfache Beziehungsmodelle:

- die Beziehung des Teils zum Ganzen (»Immer aber muß jede Einzelheit als Glied des Ganzen erscheinen. $\left.{ }^{40}\right)$

- und die von Gehalt und Gestalt (»im Kunstwerk sei alles Äußerliche ein Innerliches, und alles Innerliche erscheine, indem es nach außen tritt, als Form $\left.\ll^{41}\right)$.

Die Makroanalyse kommt nur dann ins Spiel, wenn der historische Abstand oder fehlendes Bildungswissen das intuitive Verstehen des Werks erschweren. Sie birgt jedoch die Gefahr der Grenzüberschreitung. Die »Kenntnis des Umkreises $« 42$ gilt deshalb als prekär. Trunz: »Daraus ergibt sich die Frage: Was davon soll man beim Interpretieren nennen? Möglichst wenig «43.

Auch für Benno von Wiese, der für eine stärkere literaturhistorische Orientierung der Interpretation plädiert und das »geschichtliche Wechselspiel zwischen inneren Bildungs- und Wachstumsgesetzen der Dichtung auf der einen und den Bewegungen, die von außen auf diesen Gang einwirken, auf der anderen Seite «44 betont, legt immer wieder dar, dass der Komplexitätsgrad der Dichtung, der anzeigt, in welchem Maße sie sich > Welt< angeeignet hat, nicht überboten werden darf. Er warnt davor, »Dichtung an Maßstäben zu messen, die außerhalb ihrer selbst liegen $« 45$.

Die Beschreibungen des Verhältnisses von Dichtung und Literaturwissenschaft in den 1950ern erinnern an die Versuche nachaufklärerischer Theologie, die Wissensbestände der Moderne, deren Evidenz nicht mehr bestritten werden kann, zu integrieren und die Vorstellung einer Offenbarung - alles Ursprünglichen, Substantiellen, Nichtakzidentiellen - dennoch nicht aufzugeben. ${ }^{46}$ Das »durch den Horizont des Ranghohen $\ll^{47}$ bestimmte Werk gilt in der Nachkriegsgermanistik, zumindest bei ihren führenden und den Fachdis-

38 Trunz: Über das Interpretieren deutscher Dichtung (s. Anm. 34), S. 65.

39 Trunz: Über das Interpretieren deutscher Dichtung (s. Anm. 34), S. 66.

40 Trunz: Über das Interpretieren deutscher Dichtung (s. Anm. 34), S. 66.

41 Trunz: Über das Interpretieren deutscher Dichtung (s. Anm. 34), S. 66.

42 Trunz: Über das Interpretieren deutscher Dichtung (s. Anm. 34), S. 67.

43 Trunz: Über das Interpretieren deutscher Dichtung (s. Anm. 34), S. 67. Oder auch dort zu finden: »Doch muß man damit bei einer Auslegung sparsam sein.«

44 Wiese, Benno von (Hg.): Die deutsche Lyrik. Form und Geschichte. Interpretationen. Vom Mittelalter bis zur Frühromantik. Düsseldorf 1964, S. 12.

45 Wiese: Die deutsche Lyrik (s. Anm. 44), S. 17.

$46 \mathrm{Vgl}$. die frühe Kritik an der Tendenz zur »Literaturtheologie« bei Sengle, Friedrich: »Zur Einheit von Literaturgeschichte und Literaturkritik.« In: DVjs 34 (1960), S. 327-337, hier S. 328.

47 Kayser, Wolfgang: Das sprachliche Kunstwerk. Eine Einführung in die Literaturwissenschaft. 16. Aufl. Bern 1973, S. 13. 
kurs dominierenden Vertretern als Offenbarung des Geistes und als unüberbietbare Form des Welterkennens. Deshalb vermag die Wissenschaft selbst in ihren Anfangsgründen nicht, eine Differenz zum Werk zu denken. Staiger: $»$ Schon die Methode richtet sich nach dem Gegenstand. $« 48$

Weil die historische Entwicklung der Kunst im zwanzigsten Jahrhundert selbst den von der Germanistik favorisierten Werkbegriff spätestens mit der avantgardistischen Moderne aufgehoben hat, muss die Grenze jetzt dreifach, also nicht nur im Blick auf das Wesen des Werks, auf dessen Rang, sondern auch im Blick auf seine Geschichte gezogen werden. Emil Staiger schlägt mit der »Spätzeit« einen Begriff vor, der es erlauben soll, zwischen einer Epoche, in der das Werk zu sich selbst kommen konnte, und einer Epoche des Niedergangs zu unterscheiden. Die Annahme eines Gipfels um 1800 und einer Spätzeit, die »von 1800 bis zu unseren Tagen $« 49$ reicht, erlaubt die ausschließliche Beschränkung der Literaturwissenschaft auf Werke, »die sich dankbar zur Vergangenheit bekennen oder doch davon durchdrungen sind. «50 Die drei Grenzziehungen zeigen in ihrer Kombination, dass es aus wissenschaftstheoretischer Sicht entgegen der emphatischen Behauptung von Staiger u. a. nicht der Gegenstand ist, der die Methode bestimmt, sondern dass der Gegenstand auf Grund enger Vorannahmen durch die Literaturwissenschaft konstituiert wird.

Wie ist unter derartigen Bedingungen Beobachtung noch möglich? Und was kann, soll dann überhaupt noch beobachtet werden? Das ist die Frage, die Wolfgang Kayser in seinem einflussreichen Aufriss Das sprachliche Kunstwerk zu beantworten sucht und die im Zentrum seiner Bemühungen um eine literaturtheoretische Begründung der Literaturwissenschaft steht. Den Ausgangspunkt bildet eine merkwürdige Anekdote, die Urszene einer Begegnung mit der Kunst, von der Kayser erzählt. Ein Städtebesucher ist von der Schönheit einer gotischen Kathedrale überwältigt. »Und dann erfährt man, daß es sich um einen Bau des 19. Jahrhunderts handelt. Man fühlt sich

48 Staiger, Emil: Meisterwerke deutscher Sprache aus dem neunzehnten Jahrhundert. 3. Aufl. Zürich 1957, S. 11. »Staigers Bestreben geht auf eine Art Reinigung von den historischen Schichten, die jedes einzelne literarische Zeugnis konstituieren.« So Böschenstein, Bernhard: »Emil Staigers Grundbegriffe: ihre romantischen und klassischen Ursprünge. «In: Barner/König: Zeitenwechsel (s. Anm. 27), S. 268-281, hier S. 273. "Reinigung « ist aus meiner Sicht ein metaphorischer Ausdruck für eine rigorose Komplexitätsreduktion. In diesem Zusammenhang aufschlussreich ist die Interpretation von Hölderlins »Menschenbeifall« durch Clemens Heselhaus. Dort heißt es u. a.: »Von besonderem Reiz sind bei dieser Tendenz zur Identität von Form und Geist, Metrum und Gedanke jene rhythmischen Variationen, die das Formale nicht so kalkulabel machen, daß darüber das Lebendige der Sprache ertötet würde. «Heselhaus, Clemens: »Friedrich Hölderlin. Menschenbeifall. «In: Wiese: Die deutsche Lyrik (s. Anm. 44), S. 364-368, hier S. 366.

49 Staiger, Emil: Spätzeit. Studien zur deutschen Literatur. Zürich 1973, S. 7.

50 Staiger: Spätzeit (s. Anm. 49), S. 8. Die wahren Dichter der `Spätzeit « »blicken aus Überzeugung zurück und bleiben den alten Göttern treu, vielleicht, um ihrer Wiederkehr in fernen Tagen den Weg zu bereiten, vielleicht aber auch nur, weil sie dazu bestimmt sind und nicht anders können. «Staiger: Spätzeit (s. Anm. 49), S. 12. 
beschämt und fühlt auf eine seltsame Weise den Boden unter den Füßen weggezogen. Der innere Kontakt mit dem Werk hat plötzlich ausgesetzt. « ${ }^{51}$ Es ist nicht nur der Bildungsbürger, der sich durch seinen Irrtum beschämt fühlt und einen Schock erlebt, weil er Authentisches nicht von Kopiertem zu unterscheiden vermochte und deshalb im emphatischen Sinn seine Welt nicht mehr >versteht<. »Er glaubte in dem Werk eine Botschaft zu hören und hört nun eine Lüge «52, kommentiert Kayser seine Erzählung. Hier artikuliert sich ein Nachkriegsgermanist nicht allein im Blick auf die >lügenhafte< Kunst der Moderne, sondern ebenso auf die Vergangenheit im Nationalsozialismus. Der architektonische Historismus, von dem hier die Rede ist, bediente sich - im NS-Staat auf die Spitze getrieben - der Aura des Vergangenen. Zugleich verursachte er damit deren Verlust. Von dieser Verlusterfahrung her rührt die grundsätzliche Skepsis gegenüber dem Historischen - genauer: gegenüber der vom Standpunkt der Gegenwart gedeuteten Geschichte - und den >Botschaften $<$ in der Literaturwissenschaft bei Kayser. Der ganze >Schwindel< offenbart aber gleichzeitig den prekären Beobachterstatus des modernen Geisteswissenschaftlers, der sich in der Moderne nicht mehr auf festem Grund befindet. Auf welche Weise kann er sich dann noch der Singularität und Echtheit der Kunst versichern? Welche Position muss er einnehmen, wenn die eigene Intuition und das angeeignete Bildungswissen es nicht verhindern können, dass man in die Abgründe des Unechten und Banalen stürzt?

Die Antwort derjenigen, die, wie Kayser nach 1945 nicht zufällig betont, »den eigentlichen Aufgaben treu geblieben sind «53, kann nur im Rückzug der von der Kunstmoderne (und der Politik) >Beschämten< bestehen. Ein Ort muss gefunden werden, der den »inneren Kontakt « zum Werk erlaubt und festigt. Dieser Ort ist eine Literaturwissenschaft, die »Dichtung « konsequent »als in sich geschlossenes sprachliches Gefüge « betrachtet und »die schaffenden sprachlichen Kräfte «54 erkennt. Sie sind das, was noch ohne $>$ Schwindel< beobachtet werden kann.

Um ihr Ziel zu erreichen, muss die Germanistik nach den Grenzziehungen in drei Bereichen zusätzliche Reduktionen vornehmen:

- im Historischen (»Dichtung lebt und entsteht nicht als Abglanz von irgend etwas anderem $\ll 55)$,

- in der Folge im Biographischen (»der Dichter ist in dem eigentlichen Gegenstand der Literaturwissenschaft nicht enthalten $\ll^{56}$ )

- und schließlich im Literarischen, wo es darum geht, »einen engeren Bezirk « ranghoher Dichtung »abzusondern $\ll^{57}$.

51 Kayser: Das sprachliche Kunstwerk (s. Anm. 47), S. 22.

52 Kayser: Das sprachliche Kunstwerk (s. Anm. 47), S. 22.

53 Kayser: Das sprachliche Kunstwerk (s. Anm. 47), S. 7.

54 Kayser: Das sprachliche Kunstwerk (s. Anm. 47), S. 7.

55 Kayser: Das sprachliche Kunstwerk (s. Anm. 47), S. 7.

56 Kayser: Das sprachliche Kunstwerk (s. Anm. 47), S. 17.

57 Kayser: Das sprachliche Kunstwerk (s. Anm. 47), S. 14. 
Kayser wertet die Reduktion der bisherigen Arbeitsfelder selbstbewusst als eine Innovation des Fachs: »ein neuer Abschnitt in der Geschichte der literarischen Forschung hat begonnen ${ }^{58}$. Die Konzentration auf die poetische Sprache als das »Wesen eines dichterischen Kunstwerks $«{ }^{59}$ begründet aus der Sicht Kaysers die Einheit der Germanistik neu, und zwar nun systematisch als Wissenschaft von der Sprache und Literatur. ${ }^{60}$ Auf methodischer Ebene resultiert aus dieser Systematik die Vorrangstellung einer literaturwissenschaftlichen Poetik, wie wir sie z. B. dann in Peter Szondis Theorie des modernen Dramas ${ }^{61}$ wiederfinden. Die Philologie taucht innerhalb der neuen Einheit nur noch als Hilfswissenschaft auf. ${ }^{62}$ Es zeichnet sich eine auf einen engen Bereich bezogene Disziplin ab, die von Innen nach Außen, von einem Kern, der nicht philologisch ist, zu einem nicht näher bezeichneten Rand hierarchisch geordnet ist. Auch fachsystematisch stellt die Poetik »auf jeden Fall [...] den innersten Kreis der Literaturwissenschaft dar. «63 Sie weist die größte Nähe zum Werk auf, das mit der Dichtungssprache wiederum einen eigenen »Kernbezirk besitzt, dessen Erforschung ihre eigenste und innerste Aufgabe ist. 64

Die von Kayser vorgeschlagenen, weit reichenden Reduktionen erzeugen ambivalente Wirkungen. Durch sie schließt die Literaturwissenschaft einerseits erhebliche Wissensbestände aus und verzichtet weitgehend auf Makroanalysen. Auf der anderen Seite begründet sie ansatzweise - vor dem Hintergrund zeitgenössischer organologischer und morphologischer Vorstellungen die Einheit von Sprach- und Literaturwissenschaft neu und leitet einen Rationalisierungs- und Verwissenschaftlichungsschub im Blick auf die Empirie sprachlich-poetologischer Phänomene ein. In der Terminologie Luhmanns könnte man sagen, dass Kayser von >Umweltbeobachtung< auf >Systembeobachtung< umstellt. Im Rahmen meiner noch sehr vorläufigen Untersuchung kann leider nur angedeutet werden, dass z. B. auch Eberhard Lämmerts Erzähltheorie ${ }^{65}$ und Walther Killys Lyriktheorie diese >Umstellung < mit beachtlichem Erfolg praktizieren. So lässt sich Killy bei seiner Lyrikuntersuchung davon leiten, »daß ein Gedicht, auch das einfachste, ein komplexes Gebilde darstellt, dessen Bestandteile und immanente Funktionen in einem Ganzen [...] aufgehoben sind. «66 Doch trotz der subtilen und differenzierten Ein-

58 Kayser: Das sprachliche Kunstwerk (s. Anm. 47), S. 7.

59 Kayser: Das sprachliche Kunstwerk (s. Anm. 47), S. 18.

60 Kayser: Das sprachliche Kunstwerk (s. Anm. 47), S. 18.

61 Szondi, Peter: Theorie des modernen Dramas. Frankfurt a. M. 1959.

62 Kayser: Das sprachliche Kunstwerk (s. Anm. 47), S. 24.

63 Kayser: Das sprachliche Kunstwerk (s. Anm. 47), S. 19.

64 Kayser: Das sprachliche Kunstwerk (s. Anm. 47), S. 24. Die >Kernbezirke< und >innersten Kreise< erscheinen als rettende Inseln inmitten der >bodenlosen< Moderne.

65 Vgl. Bogdal: Wissenskanon und Kanonwissen (s. Anm. 29).

66 Killy, Walther: Elemente der Lyrik. München 1983, S. 12. Auch Killy konstatiert eine >bodenlose< Zeit, die den Leser, »der in ein Zeitalter geboren ist, das keine verbindliche Poetik, ja überhaupt keine Verbindlichkeit mehr sein eigen nennt« (S. 12), orientierungslos zurücklässt. 
sichten und Erkenntnisse erscheint es auch ihm nicht möglich, den Komplexitätsgrad des Gegenstands je zu erreichen, denn »die kurze Rede des Gedichts übersteigt in ihren Möglichkeiten stets die längere des Deuters «67.

Die Nachkriegsgermanistik bedient sich, um dieses Problem zu entschärfen, immer wieder der durch den Hegelianismus, die Phänomenologie und die Existentialontologie zur Verfügung gestellten Figuren der Komplexitätsreduktion wie Teil und Ganzes, Oberfläche und Tiefe, Wesen und Erscheinung usw. Benno von Wiese legitimiert etwa die Beschränkung der Literaturwissenschaft auf das »Bedeutende« damit, dass dieses »nicht nur für sich selbst « stehe, »sondern auch stellvertretend für den Gang des Ganzen. «68 Solche Schlüsse und methodischen Operationen erscheinen heute wenig plausibel, konnten aber in den Fünfzigern in den Geisteswissenschaften noch mit Zustimmung rechnen.

\section{Komplexität und Wissen}

Die Konzentration der Auseinandersetzungen auf die werkimmanente Methode in den sechziger Jahren verdeckt zum Teil bis heute, dass die Nachkriegsgermanistik sich selbst als unübersichtlich und richtungslos wahrnimmt. Vor allem die Methodenvielfalt des geistesgeschichtlichen Erbes und das weiterhin ungeklärte Verhältnis zur Philologie des literaturwissenschaftlichen Positivismus werden als Gefährdung der Einheit betrachtet. In den Fünfzigern nehmen die Befürchtungen zu, dass das Fach sich, in erster Linie in der Älteren Philologie, in unübersichtlichen Spezialuntersuchungen im wörtlichen Sinne des Wortes $>$ verzettelt $<$. Noch 1968 fordert Jost Hermand, die Befürchtungen und Klagen resümierend: »Es ist daher unumgänglich, das Postulat des >Universalismus< aufrechtzuerhalten, und zwar trotz des stofflichen Dilemmas, trotz des naturwissenschaftlichen Exaktheitsanspruchs und trotz der steigenden Spezialisierung. «69

Der entscheidende Bruch in der Fachentwicklung ereignet sich jedoch für die Ältere und Neue Deutsche Philologie nicht voraussehbar - mit der >Entphilologisierung< der Sprachwissenschaft und der Begründung einer die Grenzen der Nationalphilologie sprengenden, empirischen und formalisierten Linguistik. Hier noch von einer >Gefährdung < der Einheit zu sprechen, wäre ein Euphemismus, denn in der Folge entstehen neue disziplinäre Ordnungen, aus denen Anfang der Siebziger das eigenständige Fach Linguistik hervorgeht, in dem als Wissenschaftssprache das Englische vorherrscht. Die ReIntegration der Linguistik bleibt das entscheidende Problem der Einheit der Germanistik, zu deren Selbstverständnis seit ihren Anfängen die Erforschung des Zusammenhangs von Sprache und Literatur gehörte. Sie stellt ein gravie-

67 Killy: Elemente der Lyrik (s. Anm. 66), S. 197.

68 Wiese: Die deutsche Lyrik (s. Anm. 44), S. 5.

69 Hermand: Synthetisches Interpretieren (s. Anm. 28), S. 169. 
renderes Problem als die kulturwissenschaftliche Wende dar, die zumindest im Blick auf die Sprachwissenschaft re-integrierende Kräfte freigesetzt hat.

Schon bei Kayser ist zu spüren, wenn er vom Literaturhistoriker »eine gründliche sprachwissenschaftliche Schulung ${ } 70$ fordert, dass die Sprachwissenschaft auf die Literaturwissenschaft wachsenden Druck ausübt, die Komplexität ihrer Mikroanalysen zu erhöhen. In den fünfziger Jahren kommt hinzu, dass Psychoanalyse, Marxismus und Soziologie eine wachsende Bedeutung in den Geistes- und Gesellschaftswissenschaften erlangen. Sie dringen zunehmend in die Domänen der Germanistik (Autor-Werk-Geschichte der Literatur) ein und deuten sie von ihren Grundlagen her als >Ganzes $<$ : als Ausdruck des Unbewussten, der Produktionsverhältnisse oder als soziales Phänomen. Sie erschließen attraktive Wissensbestände jenseits traditioneller literarischer Bildung und stellen die Germanistik vor die Anforderung, zu fachspezifischen Makroanalysen zurückzukehren. Insgesamt gerät das Fach in den Sechzigern in den Zangengriff einerseits eines seiner (ehemaligen) Teilbereiche, der einen enormen mikroanalytischen Niveaugewinn verbuchen kann, und andererseits der makroanalytischen Orientierungsleistung der aufstrebenden Gesellschaftswissenschaften.

Zur Erläuterung beschränke ich mich hier auf zwei signifikante Beispiele aus der Übergangszeit von der Nachkriegsgermanistik zur sogenannten Reformphase: einmal auf Ingrid Strohschneider-Kohrs Programmschrift Literarische Struktur und geschichtlicher Wandel. Aufriß wissenschaftsgeschichtlicher und methodologischer Probleme ${ }^{71}$, die ich an anderer Stelle ausführlich untersucht habe, ${ }^{72}$ und auf Friedrich Sengles Monumentalprojekt $»$ Biedermeierzeit $\ll .73$

In ihrer Argumentation, in der sie die beiden Entwicklungsstränge der werkimmanenten Interpretation und der geistesgeschichtlichen Literaturgeschichtsschreibung zusammenführt, arbeitet Strohschneider-Kohrs - durch Ingardens Phänomenologie und den russischen Formalismus inspiriert - als zentrales wissenschaftliches Problem die fehlende Komplexität bisheriger germanistischer Methoden heraus: »Sie sprechen nahezu alle stets nur von einer Schicht der Literatur, benennen zumeist nur ein Strukturelement des literarischen Gebildes und verfolgen monolinear von dieser von ihnen genannten Komponente her die Möglichkeit der geschichtlichen Entwicklung. Sie bezeichnen dementsprechend zumeist auch nur einen Wirkfaktor, der die Veränderung in oder von dieser Schicht her auslöst oder entstehen läßt. «74

70 Kayser: Das sprachliche Kunstwerk (s. Anm. 47), S. 19.

71 Strohschneider-Kohrs, Ingrid: Literarische Struktur und geschichtlicher Wandel. Aufriß wissenschaftsgeschichtlicher und methodologischer Probleme. München 1971.

72 Bogdal: Wissenskanon und Kanonwissen (s. Anm. 29).

73 Sengle, Friedrich: Biedermeierzeit. Deutsche Literatur im Spannungsfeld zwischen Restauration und Revolution 1815-1848. Bd. 1. Allgemeine Voraussetzungen. Richtungen. Darstellungsmittel. Stuttgart 1971.

74 Strohschneider-Kohrs: Literarische Struktur (s. Anm. 71), S. 14 f. 
Die an Kontinuität interessierte und fachpolitisch konservative Phänomenologin entwickelt in ihrem Plädoyer für die Verbindung von Gattungstypologie, Formanalyse und modernem Strukturalismus ein Bewusstsein für ein zentrales Moment moderner Wissenschaft, für die Fähigkeit nämlich, höhere Komplexität zu thematisieren. Sie zieht sich in ihrer Argumentation nicht mehr auf die Dignität des Gegenstands Literatur zurück.

Während Strohschneider-Kohrs ihr ambitioniertes Programm nicht in konkrete Forschungsprojekte umgesetzt hat, legt Friedrich Sengle 1970 mit dem ersten Band der Epochendarstellung 75 zur »Biedermeierzeit « einen literaturgeschichtlichen Entwurf vor, der in deutlichem Abstand zur werkimmanenten Methode angesiedelt wird.

Schon 1960 wendet sich Sengle in seiner Heidelberger Antrittsvorlesung kritisch gegen die von der werkimmanenten Methode und der phänomenologischen Literaturwissenschaft gleichermaßen praktizierte »radikale Trennung von Literaturgeschichte und Literaturkritik «76, die er fachgeschichtlich auf die Faszination an Gundolfs »Literaturtheologie «77 zurückführt.

In der Studie über die Biedermeierzeit nun stellt er sich auf makroanalytischer Ebene den von Strohschneider-Kohrs formulierten Ansprüchen einer vielschichtigen, nicht mehr monolinear verfahrenden Literaturgeschichtsschreibung. Zugleich verweigert er sich aus Gründen, die nur durch eine genauere fachgeschichtliche Untersuchung nachvollzogen werden können, den Ende der Sechziger diskutierten historisch-materialistischen, sozial-geschichtlichen und literaturevolutionistischen (strukturalistisch-formalistischen) Paradigmen. ${ }^{78}$ Dennoch rückt die Historizität von Literatur so weit in den Vordergrund, dass seine Darstellung, die nicht mehr einem teleologischen Entwicklungsprinzip folgen möchte, sich rasch dem Problem der Kontingenzbewältigung ausgesetzt sieht. Das Ergebnis ist ein Dualismus, der auch die späteren Sozialgeschichten der Literatur kennzeichnet: das Nebeneinander der Makroanalyse der Epoche, die von Sengle als enzyklopädischer Wissensvorrat angelegt und > vorgehalten < wird, und der nur noch partiell ausgeführten Mikroanalyse der Werke, die aus diesem Vorrat schöpfen kann.

Dennoch verzichtet Sengle nicht auf ein vereinheitlichtes, sämtliche Erscheinungen >durchdringendes< Element. Es wird zwar nicht wie in August Korffs Epochendarstellung der Goethezeit als »Geist«, sondern in einer exis-

75 Auf das Epochenproblem kann hier aus Platzgründen nicht weiter eingegangen werden. An ihm ließen sich ebenfalls die Folgen der Komplexitätserhöhung für die Erforschung von Wissensbeständen und vor allem für die Darstellung zeigen.

76 Sengle: Zur Einheit von Literaturgeschichte und Literaturkritik (s. Anm. 46), S. 328.

77 Sengle: Zur Einheit von Literaturgeschichte und Literaturkritik (s. Anm. 46), S. 328.

781960 hingegen postulierte er: »In der Geschichtswissenschaft selbst wäre die neue Wertung, und das heißt immer auch die neue Ordnung, zu suchen. Es kann in der einen [sic!] die verschiedenen Substanzen zusammenfassenden Welt, in der wir m. E. schon heute leben, nur eine bewegliche, elastische Ordnung sein. « Sengle: Zur Einheit von Literaturgeschichte und Literaturkritik (s. Anm. 46), S. 337. 
tentialontologischen, in Andeutungen soziologischen, ${ }^{79}$ Wendung als $\gg$ Grundstimmung $\ll 80$ der Epoche bezeichnet. ${ }^{81}$

Mir geht es hier nicht um die Epochenkonzeption Sengles, mit der sich die Vormärzforschung intensiv und kontrovers auseinandergesetzt hat, sondern um das Archiv, das angelegt und die Ordnung des Wissens, die präsentiert wird. Diese Ordnung erschließt sich nicht mittels der philologischen Lektüre repräsentativer oder exemplarischer Werke. Das Archiv wird angelegt, bevor noch ein einziges literarisches Werk interpretiert wird, so dass von einer Kontexterschließung nicht die Rede sein kann. Trotz der beeindruckenden Materialfülle ist die Rekonstruktion der Epoche nicht umfassend. Die >Grundstimmung < bildet eine zu unscharfe Kategorie, die außerdem stark deutende Prämissen enthält. Auf ein ereignis-, struktur- oder sozialgeschichtliches Modell greift Sengle nicht zurück, obwohl entsprechendes geschichtswissenschaftliches Wissen ständig dargeboten wird. Dessen Status wird weder im Blick auf die Epochenkonstruktion noch auf die Werkanalyse geklärt. ${ }^{82}$ Der enge Rahmen - »dämonische Zerrissenheit und sentimentaler Weltschmerz «83 als epochale Grundstimmung - erlaubt dennoch nicht eine Eingrenzung des humanwissenschaftlichen Wissens über den untersuchten Zeitraum. In auffälliger Gedrängtheit werden die politischen, sozialen und wirtschaftlichen Verhältnisse angedeutet, werden Bereiche wie Technik, Schnellpresse, Reisemode, Vereine, Frauen erwähnt, Religions- und Generationsfragen erörtert und Phänomene des Unbewussten angesprochen. ${ }^{84} \mathrm{Hinzu}$ kommen u. a. die Themen Gartenbau, Alpinismus, Körperkultur, Psychologie, Hygiene, Geschichtsschreibung, Historienmalerei und Historiendichtung, Heimat, kirchlicher Partikularismus, Eros, Familie und Mutterkult. Selbst das

79 Sengle: Biedermeierzeit (s. Anm. 73), S. 1: »Damit ist nicht gesagt, daß Lage und Stimmung wichtiger sind, als das, was man daraus macht; denn der Rang einer Generation hängt weniger von ihrer Situation als von der Bewältigung ihrer inneren und äußeren Probleme ab.«In dieser Formulierung sind durchaus Elemente der Soziologie Mannheims zu erkennen.

80 Sengle: Biedermeierzeit (s. Anm. 73), S. 1: »Wenn man wissen möchte, was die verschiedenen Ideologien, Handlungen und Gestaltungen eines Zeitraums miteinander verbindet, so wird man zunächst nach der Grundstimmung der Menschen, die in ihm leben, fragen müssen und nach der geschichtlichen Situation, die ihr entspricht.«

81 Damit nähert sich Sengle - ohne dies explizit zu machen - der zur gleichen Zeit international bekannt werdenden Mentalitätsgeschichte an.

82 Nach der Darstellung der »Grundstimmung « wird im 2. von insgesamt fünf Kapiteln des ersten Bandes die Perspektive auf das Literatursystem verengt (»Auffassung und Gebrauch der Dichtung «), das 3. differenziert die literarischen Richtungen der Zeit, das 4., aus meiner Sicht innovativste, untersucht »Symbol, Begriffsallegorie, Naturpersonifikation. Mythologie« und das sehr ausführliche 5. ganz im Sinne der Hauptlinie der Nachkriegsgermanistik die Literatursprache vom Wortschatz bis zum Stil. Es kommt damit der Forderung nach einer wissenschaftlichen Poetik nach.

83 Sengle: Biedermeierzeit (s. Anm. 73), S. 2.

84 Sengle: Biedermeierzeit (s. Anm. 73), S. 1: »Aber das geschichtliche Verständnis einer Epoche ist ohne den Vorstoß zu ihren geheimeren und oft unbewußten >Gründen nur sehr unvollkommen möglich.« 
»Jahrbuch für Deutschlands Heilquellen «85 und die »Cholera von 1831 «86 sind einer Erwähnung wert. ${ }^{87}$

Jedes der durch den Begriff der »Grundstimmung « nur lose verknüpften Themen müsste zu einer kulturwissenschaftlichen Studie nach heutigem Sprachgebrauch ausgeweitet werden, sobald den Text-Kontext-Relationen konkret nachgegangen würde.

Die >Entgrenzung « wird also schon vor mehr als 30 Jahren bei Sengle zu einem Forschungs- und Darstellungsproblem, das sich in dem Augenblick (wieder) einstellt, wenn man sich in Theorie und Praxis der Beschreibung von Kontingenz und Komplexität historischer Prozesse widmet.

\section{Komplexität und Einheit?}

Die Programmschrift von Strohschneider-Kohrs und Sengles Epochendarstellung 88 offenbaren die Konsequenzen der >Modernisierung < des Fachs. Die angestrebte Komplexität ist weder in einem einzigen theoretischen Modell abbildbar, noch lässt sie sich materialiter von einem einzelnen Forscher meistern. Dennoch werden die von Strohschneider-Kohrs auf der einen und von Sengle auf der anderen Seite eingeschlagenen Wege weiter begangen, was sich an den Methoden- und Theoriedebatten und an der Entwicklung einer Sozialgeschichtsschreibung der Literatur zeigen ließe.

Jede fachgeschichtliche Beschreibung sieht sich vor die Schwierigkeit gestellt, dass in dieser Phase, in der sich konkurrierende Theorieangebote und Forschungsprogramme bis zur Unübersichtlichkeit vervielfältigen, zugleich jeweils auch der Zuschnitt der Disziplin mit konzipiert wird.

Deshalb werde ich für die weitere Darstellung hilfsweise auf signifikante Quellen zurückgreifen, die trotz disparater Elemente gleichermaßen Theorien, Forschungsstand und disziplinäre Ordnung repräsentieren. An ihnen hoffe ich exemplarisch zeigen zu können, wie die >Modernisierung < zu unterschiedlichen Modellen von >Einheit $<$ geführt hat.

\section{Die Einheit des Katalogs}

Wenn man akzeptiert, dass die von Wolfgang Stammler herausgegebene dreibändige Deutsche Philologie im Aufriß, die in der ersten Auflage zwischen 1952 und 1957, in der zweiten zwischen 1957 und 1962 erscheint, einen weit-

85 Sengle: Biedermeierzeit (s. Anm. 73), S. 39.

86 Sengle: Biedermeierzeit (s. Anm. 73), S. 40.

87 Insofern lässt sich Sengles Epochendarstellung, zumal wenn man die zahlreichen, von ihm angeregten Dissertationen hinzunimmt, als Vorläufer geisteswissenschaftlicher Großforschungsprojekte betrachten.

88 Auf eine breite Auswertung vergleichbarer germanistischer Arbeiten des Zeitraums muss aus Darstellungsgründen verzichtet werden. 
hin akzeptierten Versuch darstellt, die Germanistik unter dem traditionellen Titel Philologie

- als einheitliches Fach zu repräsentieren,

- die innere Systematik

- und den Wissensstand bzw. Forschungsstand darzustellen,

ließe sich an ihr der Entwicklungsstand der 1950er zureichend erfassen. ${ }^{89}$ Der »Aufriß« gliedert die Deutsche Philologie in fünf Abteilungen »Methodenlehre $\ll(1)$, »Sprachgeschichte und Mundarten« (2), »Kulturkunde und Religionsgeschichte $(4)$, »Volkskunde « (5) und die dritte Abteilung ohne Gesamtüberschrift in die Abschnitte »Literaturgeschichte in Längsschnitten«, »Ausländische Einflüsse«, »Sprachkunst in Wirkung und Austausch« und »Der Dichter hat das Wort.« Sämtliche Gebiete, die an Germanischen bzw. Germanistischen Seminaren in Deutschland gelehrt werden, sollen erfasst werden. Im »Aufriß«, dessen Vollständigkeit im Wesentlichen durch Addition erreicht wird, waltet an keiner Stelle ein philologischer Purismus. Er gleicht vielmehr, wenn man die detaillierten Unterkapitel hinzuzieht, dem heute beklagten Zustand eines entgrenzten, konturlosen Fachs. Der Aufbau lässt nur eine geringe Systemlogik erkennen, er erinnert bisweilen an Borges' $>$ gewisse chinesische Enzyklopädie <. Die Demutsgeste der Wissenschaft gegenüber der Kunst fehlt nicht. In einem eigenen Kapitel kommen u. a. Werner Bergengruen und Bernt von Heiseler zu Wort, bzw. >haben< es, was bei Dichtern so sein sollte. Im Blick auf Systematik und Kohärenz lassen sich zahlreiche Fragen stellen. Welcher Methodenbegriff verbindet »Poetik«, »Stoffund Motivgeschichte « mit der »Inschriftenkunde« und »Paläographie « $\mathrm{zu}$ einer Einheit? Weshalb nehmen Gebiete wie »Deutsche Sprache im Ausland «, »Sprechkunde« oder »Pennsilfaanisch« einen eigenen Platz ein, bilden »Ausländische Einflüsse« gar eine Unterabteilung von mehr als 600 Seiten? Abschnitte wie »Amerika im deutschen Dichten und Denken« und »Orient und deutsche Literatur « sind von gegenwärtigen kulturwissenschaftlichen Forschungsprojekten nicht weit entfernt. Überraschend tauchen als Fachgebiete der Deutschen Philologie auch »Zeitung und Zeitschrift «, der »Rundfunk « und der »Film« auf. Die IV. Abteilung, »Kulturkunde und Religionsgeschichte behandelt u. a. »altgermanische Religionen«, »Grundzüge der katholischen Kirchengeschichte «, »Geschichtsschreibung im Mittelalter «, »neuere deutsche Geschichtsschreibung «, »deutsche Verskunst« (diese also nicht unter Methodenlehre) und in Grimmscher Tradition »deutsches Recht «. Die Abteilung »Volkskunde«steht, was den thematischen Einfallsreichtum betrifft, heutiger Kulturwissenschaft in Nichts nach. So steuert der Herausgeber Wolfgang Stammler selbst einen Beitrag über »Seemanns Brauch und Glaube « bei und beschließt den »Aufriß«mit einem Artikel von Josef Göhler über »Leibesübungen in der deutschen Sprache und Literatur«. Die Herkunft nicht weniger

89 Nach vorne weisende Entwicklungen, die dort der Repräsentativität der Darstellung zum Opfer fallen, können auf diese Weise nicht aufgespürt werden. 
Beiträge lässt nur allzu deutlich erkennen, dass der Höhepunkt des betreffenden Forschungsgebiets zwischen 1933 und 1945 lag. Doch ist hier Ideologiekritik nicht intendiert. Es geht um die Systematik unseres Fachs, das sich in einer repräsentativen Selbstdarstellung ein letztes Mal als Deutsche Philologie bezeichnet. Der »Aufriß« wahrt dessen Einheit, in dem er - manchmal nur noch über das Nebenlehrgebiet eines Ordinarius oder Extraordinarius institutionell tradierte Teilgebiete der Germanistik abbildet. Für eine innere Systematik oder das Bemühen um eine einheitliche Fachterminologie gibt es keinerlei Anzeichen. Einer wissenschaftstheoretischen Kritik hätte diese Konstruktion schon in den Fünfzigern nicht standhalten können; in den Sechzigern bricht sie unter den ersten kritischen Einwänden zusammen. Mit ihr verschwindet der Begriff der Philologie als Rahmenbezeichnung der Germanistik. Während die Fachsystematik, die der »Aufriß « überliefert, bei jenen Universitätsgründungen, die wie im Falle Bochums in der ersten Hälfte der Sechziger erfolgten, zunächst noch eine Rolle spielt, setzten sich spätere Neugründungen wie Bielefeld, Konstanz oder Bremen bewusst davon ab. ${ }^{90}$ Erhalten bleibt an verschiedenen Universitäten die institutionelle Tradition in den Bezeichnungen der Institute und Lehrstühle.

\section{Einheit durch Gegenstandsbestimmung}

Die 1973 erschienenen, von Heinz Ludwig Arnold und Volker Sinemus herausgegebenen, Grundzüge der Literatur- und Sprachwissenschaft präsentieren eine Neuordnung des Fachwissens, die sich deutlich von der Deutschen Philologie im Aufriß unterscheidet. ${ }^{91}$ Für unseren Diskussionszusammenhang ist von Belang, dass der Begriff >Philologie< als Rahmenkonzept verschwunden ist. Doch nicht nur das. Er taucht nur noch an einer einzigen Stelle - und dort im Plural - auf, um an die historische Herkunft zu erinnern. Das Gesamtkonzept bindet das neue Fach Linguistik und die Literaturwissenschaft zusammen, die Ältere Deutsche Philologie findet keinen Platz mehr.92 Das liegt u. a. darin begründet, dass der Band nicht auf die Germanistik beschränkt, sondern fächerübergreifend angelegt ist. Doch in den anderen Philologien wie der Anglistik, Romanistik, Slawistik usw. nimmt die Erforschung älterer Sprachstufen und früher Literaturentwicklungen zu dieser Zeit noch einen breiten Raum ein und hätte im Rahmen der universalisierenden, von einzelnen

90 An dieser Stelle müsste eine präzise Analyse der Weiterentwicklung der im »Aufriß« genannten Teilgebiete stehen: der Umorganisierung, des Verschwindens, der Ausgliederung wie bei der Volkskunde, die durch die kulturwissenschaftliche Wende eine partielle Re-Integration erfährt, der Profilierung der AVL usw. In diesem Rahmen müssen erste Hinweise und Andeutungen genügen.

91 Der Band ist wie folgt gegliedert: »Textwissenschaft«; »Strukturelle Textwissenschaft «; »Textkritik und Edition«; »Grundlagen des Textverständnisses«; »Grundlagen der Textgestaltung «; »Bauelemente von Textarten«; »Textarten«; »Methoden der Textanalyse «; »Unterhaltungs- und Trivialliteratur «.

92 So weit ich das eruieren konnte, arbeitete kein Mediävist an den »Grundzügen« mit. 
nationalen Literaturen und Sprachen absehenden Darstellung einen Ort finden können. ${ }^{93}$

Bei genauerer Durchsicht fällt auf, dass die Beiträger der »Grundzüge « im Inhaltsverzeichnis nicht namentlich genannt werden und ein Mitarbeiterverzeichnis fehlt. Die anonymisierende Präsentation deute ich als Ausdruck eines veränderten, in den Geisteswissenschaften vorher nicht akzeptierten szientistischen Wissenschaftsverständnisses. ${ }^{94}$ In der stark veränderten, nun von Heinz Ludwig Arnold und Heinrich Detering herausgegebenen Neuauflage von 1996 können die Verfasser an beiden Orten identifiziert werden: auch dies ein (kleines) Zeichen erneuten Wandels.

Wenn man einer Betrachtung die Kriterien Übersichtlichkeit, Systematik und Einheitlichkeit zu Grunde legt, sind die »Grundzüge « dem »Aufriß« deutlich überlegen. Die Systematik wird aus einem linguistisch fundierten, literaturwissenschaftlich erweiterten Textbegriff entfaltet. Die stringente Ableitung erlaubt eine klarere disziplinäre Grenzziehung als die in der »Deutschen Philologie « vorgenommene - und dies trotz des Eingeständnisses der Herausgeber, »daß über die Kriterien für die Wissenschaftlichkeit der Literaturwissenschaft zur Zeit keinerlei Übereinstimmung erreichbar zu sein scheint: weder über den Gegenstand, über den sie Erkenntnisse formuliert, noch über die Methoden, die sie dabei verwendet, noch über das Erkenntnisinteresse, das sie dabei leitet. «95 Für die Wissenschaftlichkeit, »die den Ansprüchen moderner Wissenschaftstheorie zu genügen mag « ${ }^{96}$, wie explizit angemerkt wird, stehen nicht mehr die philologischen Tugenden ein, sondern, auch für die Literaturwissenschaft, der sprachwissenschaftliche Textbegriff. Doch durch die Konzentration auf den Textbegriff entfernen sich die »Grundzüge« entgegen der im Vorwort der Herausgeber artikulierten Intention nur unwesentlich vom Werkbegriff der philologischen Tradition. Deshalb sind die »Grundzüge« aus meiner Sicht ein Dokument des Übergangs und fachgeschichtlich der Programmschrift Ingrid Strohschneider-Kohrs' und Sengles Epochendarstellung zuzuordnen.

Allerdings unterbreiten sie über den in dieser Phase wissenschaftstheoretisch nicht mehr hintergehbaren Weg der Gegenstandsbestimmung ein ReIntegrationsmodell für die zerbrochene - disziplinäre, nicht fachliche - Einheit von Linguistik und Literaturwissenschaft. Paradoxerweise ist dieses Modell innerhalb der Literaturwissenschaft deshalb wenig konsensfähig, weil es mit der neuen Gegenstandskonstruktion zugleich einen anderen Wissenschaftsstil und ein anderes Wissenschaftsverständnis verbindet. ${ }^{97}$

93 Hier taucht ein wichtiger Aspekt der disziplinären Entwicklung auf, dem ich aus Darstellungsgründen leider nicht weiter nachgehen kann.

94 Und dem Verzicht auf die Autorität und Reputation der Beteiligten.

95 Arnold, Heinz Ludwig/Sinemus, Volker (Hg.): Grundzüge der Literatur- und Sprachwissenschaft. Bd. 1. Literaturwissenschaft. München 1973, S. 8.

96 Arnold/Sinemus: Grundzüge der Literatur- und Sprachwissenschaft (s. Anm. 95), S. 9.

97 Vgl. Bogdal: Wissenskanon und Kanonswissen (s. Anm. 29) zu Jens Ihwe und S. J. Schmidt. 


\section{Einheit durch lebensweltliche Einbettung}

Das von Helmut Brackert und Eberhard Lämmert 1977 und 1978 in zwei Bänden publizierte Funk-Kolleg Literatur stellt keinen wissenschaftsinternen Versuch der Ordnung des Wissens über Literatur dar, obwohl die Beiträger zu den bekanntesten Vertretern ihres Fachs gehören. Es ist auch »kein Aufriß einer Einzelphilologie «98, wie es in der Einleitung heißt. Obwohl Brackert in Frankfurt am Main >Deutsche Philologie< lehrt, bleibt die ältere deutsche Literatur und mit ihr die Altgermanistik außerhalb der vom »Funk-Kolleg« gezogenen Grenzen.

Dennoch bietet das »Funk-Kolleg« ebenso eine Gesamtschau wie die »Grundzüge «. Das gleiche Prinzip der Interdisziplinarität, ${ }^{99}$ das im Kontext der Modernisierungen wissenschaftlichen Fortschritt signalisiert, zeitigt unterschiedliche Konsequenzen. Während es in den »Grundzügen« zu einer Neuformierung der Forschung auf einer universelleren Ebene führt, sind im »Funk-Kolleg« Anzeichen der >Entgrenzung « nicht zu übersehen.

Das Kolleg setzt »bei den alltäglichen Formen des Umgangs mit Literatur $\ll 100$ an. Damit folgt es sowohl einer funktionalen Betrachtungsweise als auch der Rezeptionsästhetik und der Literatursoziologie, also Richtungen, die seit Ende der Sechziger in den Mittelpunkt der Fachdebatten rücken. Das als lebensweltlich zu bezeichnende Konzept ist offen, aber keinesfalls >entgrenzend im Sinne der Kritik an der neueren Kulturwissenschaft, denn im Zentrum steht die >Literatur< als soziales, historisches und ästhetisches Phänomen. Die vorgestellte >Einheit<, die von den grundlegenden Fähigkeiten des Lesens und Schreibens bis zur Literaturgeschichte ${ }^{101}$ reicht, ist eine des kulturellen Gegenstands Literatur und unseres Wissens darüber.

Die Herausgeber und Autoren distanzieren sich bewusst von einer akademischen, von Fachsystematik und Spezialforschung bestimmten Betrachtungsweise und sehen in der Leistung der Wissenschaft für die Gesellschaft den entscheidenden Zugewinn. Aber mit dem pädagogischen Impetus, Kenntnisse, Einsichten und Fähigkeiten elaborierten Lesens zu vermitteln, ist das Kolleg wiederum näher bei der Pädagogik der neuhumanistischen Philologie des 19 . Jahrhunderts ${ }^{102}$ als die $»$ Grundzüge « mit ihrer Wissenschaftshermetik.

98 Brackert, Helmut/Lämmert, Eberhard (Hg.): Funkkolleg Literatur 1. Frankfurt a. M. 1977, S. 12.

99 Vgl. Voßkamp, Willhelm: »Grenzüberschreitungen. Zur Entstehung und Konzeption des Bielefelder >Zentrum für interdisziplinäre Forschung «« In: Bogdal/Müller: Innovation und Modernisierung (s. Anm. 24).

100 Brackert/Lämmert: Funkkolleg Literatur 1 (s. Anm. 98), S. 10.

101 Das Kolleg enthält folgende Lerneinheiten: »Lesen und Schreiben«; »Literatur - Medium der Kommunikation«; »Textkonstitution«; »Textauslegung«; »Wer schreibt, wer verbreitet, wer liest Literatur?«; »Literaturgeschichte«; »Literaturkritik und Literaturwissenschaft«; »Was ist, was soll, was kann Literatur «.

102 Zur Pädagogik der Philologie siehe Gumbrecht: Die Macht der Philologie (s. Anm. 20), S. $109 \mathrm{ff}$. 


\section{Einheit durch Selbstreflexion}

In der grundlegend umgearbeiteten Neuauflage des Kollegs, die 1992, nun von Helmut Brackert zusammen mit Jörn Stückrath herausgegeben, unter dem Titel Literaturwissenschaft. Ein Grundkurs in »rowohlts enzyklopadie« erscheint, wird die lebensweltliche Orientierung aufgegeben. Das Werk wird nun gemäß der veränderten Zielgruppe an fachwissenschaftlichen Ordnungsmustern ausgerichtet. ${ }^{103}$

Die Altgermanistik erhält zwar auch hier keinen eigenen Ort innerhalb des neuen Systems, aber mediävistische Themen tauchen in mehreren Kapiteln an wichtigen Stellen auf: eine Werkinterpretation von Wolframs Willehalm des Mitherausgebers Helmut Brackert, Beiträge über »Probleme der Mündlichkeit und die Anfänge der deutschen Literatur « und über »Schriftlichkeit und Mündlichkeit im Hoch- und Spätmittelalter « und eine literaturtheoretische Positionsbestimmung von Werner Röcke zur »Literaturgeschichte - Mentalitätsgeschichte «.

Die Rückkehr der > $̈$ Älteren Deutschen Philologie $<$ in einen auf einen breiten Konsens berechneten Grundkurs, der den Kernbestand der Literaturwissenschaft zu präsentieren beabsichtigt, ist ein deutliches Symptom für eine disziplinäre Re-Integration. Diese Re-Integration bedeutet aber zugleich eine weitere Komplexitätserhöhung, da die neue Mediävistik sich kulturwissenschaftlich orientiert und - ein Beispiel von vielen - nun auch im Blick auf die neuere und neueste Literatur Phänomene >längerer Dauer $<$ mitgedacht werden müssen.

Wenn man nach den Ursachen für die >Rückkehr $<$ zunächst bestimmter Themen und Forschungsperspektiven sucht, so stößt man darauf, dass die Mediävistik seit den achtziger Jahren auf innovative Weise wichtige Forschungsfelder besetzt und -ergebnisse erbracht hat, die wie Schriftlichkeit/Mündlichkeit oder die Memoriaforschung von allgemeinerem Erklärungswert sind. Diese konkreten Leistungen waren entscheidend für die Re-Integration und die dann einsetzende breite Kommunikation und nicht die Frage, ob damit eine Re-Philologisierung betrieben oder eine disparate Kulturwissenschaft befördert wurde.

Im »Grundkurs« von 1992 wird die Einheitlichkeit weder durch den Gegenstand noch über seine lebensweltlichen Funktionen hergestellt, wie das Beispiel der Älteren Deutschen Philologie zeigt. Angestrebt wird sie hier durch Theoriebewusstsein, fachgeschichtliche Reflexion und interdisziplinäre >Anschlussfähigkeit<. >Einheit< erhöht also den Grad der Komplexität ein weiteres Mal und die Komplexitätserhöhung signalisiert zugleich den >Fortschritt< gegenüber dem ersten Konzept. Die »Funk-Kolleg«-Version von 1977/78 und den »Grundkurs« von 1992 eint die Vorstellung von Wissenschaft und Wissen

103 Gliederung des Grundkurses: »Grundbegriffe der Texterschließung «; »Beispiele der Texthermeneutik«; »Literarische Typenbildung «; »Realisierungsformen des Literarischen« (u. a. Probleme der Mündlichkeit, Schriftlichkeit u. Mündlichkeit, Theater, Fernsehen); »Literarische Institutionen «; »Grundfragen der Literaturwissenschaft «; »Zur Geschichte der Literaturwissenschaft «; »Aktuelle Positionen«. 
als Kommunikation, Dialog und >Leistung < im Blick auf andere Systeme, ein handlungstheoretisches Grundverständnis ${ }^{104}$ also, das sich in der Germanistik der Nachreformphase weitgehend durchgesetzt hat.

Der Blick auf die Fachgeschichte erlaubt erste vorläufige Schlussfolgerungen, die für die aktuelle Positionsbestimmung und die Debatte um die Grenzen der Germanistik von Belang sind. Die immer wieder vorgebrachte These, dass die Germanistik durch den Bruch mit der philologischen-geistesgeschichtlichen Tradition, der sich am linguistic turn, der Rückkehr zur Geschichte, dem erweiterten Gegenstandsbereich und einem politisch-gesellschaftlichen Selbstverständnis beobachten lasse, ihre Einheit in Gefahr gebracht habe, ist mit den fachhistorischen Befunden nicht in Einklang zu bringen. Insbesondere ist die Bedeutung des philologischen Kerns nicht erst in der Reformphase relativiert worden. Es hat im Gegenteil auch in den 1960ern ernsthafte Versuche gegeben, auf die Anfänge des Fachs und der modernen Hermeneutik um 1800 zurückzugreifen und eine eigenständige »philologische Erkenntnis « von anderen wissenschaftlichen Zugängen zu unterscheiden. ${ }^{105}$

Eine Bruchlinie verläuft aus wissenschaftstheoretischer Sicht zwischen der vormodernen Vorstellung von Wissenschaftssystematik und disziplinärer Einheit, wie sie sich in der Deutschen Philologie im Aufri $\beta$ widerspiegelt, aber auch noch in dem positivistischen Konzept der »Grundzüge « auf der einen, und dem handlungstheoretischen Grundverständnis auf der anderen Seite, wie es sich in den beiden genannten Beispielen, dem »Funk-Kolleg « und dem »Grundkurs « äußert.

Für die Fachentwicklung nach 1945 konnten einige Modelle herausgearbeitet werden, die das Verhältnis von Komplexitätsbewältigung und Facheinheit auf unterschiedliche Weise geregelt haben. Sie lösen sich historisch ab und erzeugen auf diese Weise eine Bewegung in der Zeit, die in der Regel als $>$ Fortschritt $<$ wahrgenommen worden ist. Ob sie Transformationen der Systematik, der Theorien, der Gegenstände und der Methoden bewirkt haben, ist damit noch nicht erwiesen. Diese Frage zu beantworten, gehört zu den zentralen Aufgaben der Fachgeschichtsschreibung.

Mit ihrer Verdrängung aus dem Zentrum des Fachs verschwinden die jeweiligen Modelle - und weitere, bei differenzierterer Erforschung zu entdeckende - nicht; sie werden nicht nur als Randpositionen in unterschiedlicher Stärke tradiert, sondern sie bleiben in den Forschungsergebnissen, deren Rahmen sie einst gebildet haben, bis heute präsent und können, wie in den jüngsten Debatten, wieder abgerufen werden. Ihr Vergleich vermittelt die Einsicht, dass die Reduktion von Komplexität nicht zur Einheit des Fachs

104 Für beide Versionen ließe sich Habermas' Theorie der kommunikativen Kompetenz als erkenntnistheoretischer Hintergrund nachweisen.

105 Vgl. Szondi, Peter: Hölderlin-Studien. Mit einem Traktat über philologische Erkenntnis. Frankfurt a. M. 1970 und König, Christoph: »Kritische Philologie heute«. In: Schönert, Jörg (Hg.): Literaturwissenschaft und Wissenschaftsforschung. DFG Symposium 1998. Stuttgart u. a. 2000, S. 317-335. 
zurückführt. Sie bewirkt im Gegenteil durch neue Grenzziehungen Ausschlüsse, wie sich am Beispiel der Volkskunde zeigen ließe, für die in den 1970ern als ein an materiellen Dokumenten interessiertes Fachgebiet innerhalb einer textorientierten Germanistik wenig Raum blieb. Umgekehrt zeigt der Vergleich auch, dass >Entgrenzungen< dennoch unterschiedliche Konzeptionen disziplinärer Einheit erlauben. Diese Situation erfordert allerdings einen höheren Grad an wissenschaftlicher Kommunikation, vernetzter Forschung, Theoriebewusstheit, Selbstreflexion und Verständigung über Organisation, Institution und Leistung des Fachs.

\section{Für Einheit und Komplexität}

In den 1960ern steckt die »Emphase der Moderne «106 auch die Germanistik an. Das Fach wird von der gesellschaftlichen Entwicklung auf verschiedenen Wegen eingeholt: über die Expansion zum Massenfach in einer Massenuniversität, über die universitäre Deutschlehrerausbildung, über die Dominanz eines szientistischen Wissenschaftsbegriffs und nicht zuletzt über den Glauben an die emanzipative bzw. demokratisierende Funktion von Literatur und Sprache und ihrer Wissenschaften usw. Modernisierungstheoretiker haben darauf hingewiesen, dass die Nachkriegszeit von einem Evolutionsdenken charakterisiert ist, das den >Fortschritt< zur Leitidee jeglicher Entwicklung erklärte. ${ }^{107}$ Andere Vorstellungen über historische, soziale, ökonomische und wissenschaftliche Prozessabläufe geraten in den Hintergrund, ${ }^{108}$ während dem Zusammenhang von »Komplexität und Problemlösungsfähigkeit «109 eine immer stärkere Schlüsselstellung zufällt.

In Forschungsprozessen gilt nun eine »zunehmende Wahrnehmungsoffenheit [...] kraft Informations- und Kommunikationserhöhung «110 als Anzeichen der erwünschten Modernität. Die sich zur gleichen Zeit auf den >inneren Kern< von Sprache und Literatur beschränkende Germanistik musste als Wissenschaft erscheinen, ${ }^{111}$ die den Anforderungen der Zeit nicht gewachsen

106 Mergel, Thomas: »Geht es weiter voran? Die Modernisierungstheorie auf dem Weg zu einer Theorie der Moderne.«In: ders./Welskopp, Thomas (Hg.): Geschichte zwischen Kultur und Gesellschaft. Beiträge zur Theoriedebatte. München 1997, S. 203-232, hier S. 216.

107 Mergel: Geht es weiter voran? Die Modernisierungstheorie (s. Anm. 106), S. 216 f.

108 Vgl. Mergel: Geht es weiter voran? Die Modernisierungstheorie (s. Anm. 106), S. 217.

109 Mergel: Geht es weiter voran? Die Modernisierungstheorie (s. Anm. 106), S. 217.

110 Mergel: Geht es weiter voran? Die Modernisierungstheorie (s. Anm. 106), S. 217.

111 Vgl. Wegmann: »Philologische Selbstreflextion.« In: Fohrmann/Voßkamp: Wissenschaft und Nation (s. Anm. 4), S. 120: »Die Differenz zwischen einer fachspezifischen Begrifflichkeit und dem Gegenstand wird demnach gerade nicht betont. Entscheidend sind vielmehr die gegenstandsbezogenen Qualitäten wie Größe, Erhabenheit, Vorbildcharakter etc. In ihnen manifestiert sich der Bildungswert des Objekts, von ihm her motiviert und rechtfertigt sich die philologische Arbeit.« 
ist, vor allem dann, wenn sich in den Natur- und Gesellschaftswissenschaften die Auffassung durchsetzt, dass »erhöhte Komplexität mit erhöhter Problemlösungsfähigkeit «112 unmittelbar zusammenhängt. Diese Feststellungen zielen nicht auf eine Übernahme modernisierungstheoretischer Konzepte. Sie sind als historische Befunde zu verstehen. Für die sich im universitären Bereich Ende der Sechziger durchsetzenden Modernisierungstheorien ist die Komplexitätserhöhung das entscheidende Element, um wissenschaftlichen Fortschritt zu denken und zu bewerten. ${ }^{113}$

Welche Position nimmt die Nachkriegsgermanistik in diesem Zusammenhang ein? Eine widersprüchliche: Sie bewegt sich mit ihrer impliziten und expliziten Literaturtheorie, um noch einmal hilfsweise Luhmanns Terminologie zu übernehmen, im Bereich »unbestimmte(r)/unbestimmbare(r) Systemkomplexität (Bereich latenter Strukturen und Prozesse) «114. In ihrer edierenden, kommentierenden, interpretierenden und strukturanalytischen Praxis hingegen stellt sie eine »bestimmte/bestimmbare Systemkomplexität (Bereich manifester Strukturen und Prozesse) $\ll 115$ dar. Die gleiche Widersprüchlichkeit gilt für die »Umweltkomplexität«, d. h. für die interpretatorische Kontextanalyse und die Literaturgeschichtsschreibung.

Der Autoritätsverlust der Nachkriegsgermanistik resultiert daraus, dass der Bereich des >Unbestimmbaren<, der mit dem Gegenstand, der Dichtung, gleichgesetzt wird, die Germanistik begründen, legitimieren und von anderen Wissenschaften unterscheiden soll. Der Bereich des >Bestimmbaren $<$, d. h. der konkrete Untersuchungsgegenstand, wird hingegen als sekundär betrachtet.

Dieses Verhältnis wird in den Fachdebatten und Forschungsprogrammen seit Ende der Fünfziger Jahre Schritt für Schritt umgekehrt, wie ich exemplarisch zu zeigen versucht habe. Diese Umkehrung kann - aus modernisierungstheoretischer Perspektive - als Verwissenschaftlichung der Germanistik beschrieben werden, die durch »Komplexitätserweiterung «116 nun dazu gezwungen ist, sich mit kontingenten Prozessen auseinander zu setzen und diese theoretisch zu modellieren. Dieser von nicht mehr abreißenden Selbstverständigungsdebatten begleitete und bis heute andauernde Prozess verlief in konjunkturellen Zyklen und gelangte niemals zu einem paradigmafähigen Ergebnis. Wenn weiterhin gilt, dass die »Komplexität des Wissens [...] Pluralität der Hypothesen, der Methoden und der Disziplinen «117 erfordert, wird sich dies auch in Zukunft nicht ändern.

112 Mergel: Geht es weiter voran? Die Modernisierungstheorie (s. Anm. 106), S. 217.

113 Vgl. Luhmann, Niklas: »Systemtheoretische Argumentation«. In: ders./Habermas, Jürgen: Theorie der Gesellschaft oder Sozialtechnologie - Was leistet die Systemforschung. Frankfurt a. M. 1971, S. 291-377, hier 292 ff.

114 Luhmann: Systemtheoretische Argumentation (s. Anm. 113), S. 301.

115 Luhmann: Systemtheoretische Argumentation (s. Anm. 113), S. 301.

116 Luhmann: Systemtheoretische Argumentation (s. Anm. 113), S. 313.

117 Rippl, Daniela/Ruhnau, Eva: »Vorwort«. In: dies. (Hg.): Wissen im 21. Jahrhundert. Komplexität und Reduktion. München 2002, S. 7-10, hier S. 7. 